\title{
Suture Tape-Reinforced Human Dermal Allograft Used for Superior Capsule Reconstruction Demonstrates Improved Ability to Withstand Elongation
}

\author{
Cody S. Lee, M.D., Manoj Reddy, M.D., Bryan Scott, M.D., Daniel Curtis, M.D., \\ Farid Amirouche, Ph.D., and Aravind Athiviraham, M.D.
}

Purpose: To investigate the potential for a suture tape-reinforcement technique to prevent elongation with repetitive use of a human dermal (HD) allograft traditionally used for superior capsule reconstruction in a biomechanical model. Methods: Using 8 scapulae and humeri Sawbones models, the standard superior capsule reconstruction was performed using $8 \mathrm{HD}$ allografts. Four grafts were tested in the native state, whereas 4 were tested using reinforcement. Allografts were reinforced using suture tape in a running $360^{\circ}$ fashion around the allograft borders, maintaining $5 \mathrm{~mm}$ of space from the edges. Allografts were measured pre- and postdynamic testing for length, width, and thickness. All specimens were affixed to a materials testing machine that allowed for allograft orientation in a longitudinal plane throughout testing. Specimens were preloaded to $10 \mathrm{~N}$ and then cyclically loaded to $100 \mathrm{~N}$ at a rate of $15 \mathrm{~mm} / \mathrm{s}$ for 30 cycles. Results: After dynamic, cyclic loading, suture tape-reinforced allografts experienced a significantly smaller percent change in anterior length $(6.36 \%$ vs $14.50 \%, P=.013)$, posterior length $(6.00 \%$ vs $13.68 \%, P=.002)$, medial width $(5.80 \%$ vs $21.05 \%, P=$ $.001)$, lateral width $(5.45 \%$ vs $19.29 \%, P<.001)$, medial thickness $(4.38 \%$ vs $17.93 \%, P=.005)$, central thickness $(7.03 \%$ vs $16.11 \%, P=.026)$, and lateral thickness $(4.55 \%$ vs $20.80 \%, P<.001)$. Linear stiffness values obtained for suture tape -reinforced allografts were significantly greater than those for native allografts when measured at cycles 1 $(21.18 \pm 1.03 \mathrm{~N} / \mathrm{mm}$ vs $17.69 \pm 1.91 \mathrm{~N} / \mathrm{mm}, P=.02), 15(29.90 \pm 1.45$ vs $24.93 \pm 2.79, P=.03)$, and $30(32.13 \pm 1.98 \mathrm{~N} /$ $\mathrm{mm}$ vs $25.72 \pm 3.01 \mathrm{~N} / \mathrm{mm}, P=.01)$ of dynamic testing Conclusions: The suture tape-reinforcement technique described decreased HD allograft elongation, maintained graft thickness, and improved linear stiffness values following uniplanar cyclic loading in a biomechanical Sawbones model. Clinical relevance: Suture tape reinforcement of the HD allograft could serve as one strategy to overcome the issue of allograft elongation with time following superior capsule reconstruction.

S uperior capsule reconstruction (SCR) of the shoulder represents a promising treatment option for patients with massive, irreparable rotator cuff tears and minimal glenohumeral degenerative changes who are not interested or otherwise candidates for reverse total shoulder arthroplasty. ${ }^{1-3}$ Despite advances through biomechanical investigations, important questions remain regarding the optimal graft material and its properties. ${ }^{3-5}$ In the original description of SCR, Mihata et al. $^{2,3}$ demonstrated excellent biomechanical and clinical results using fascia lata (FL) autograft. In subsequent biomechanical studies, Mihata et al. ${ }^{4-7}$ demonstrated improved characteristics of FL autograft compared with human dermal (HD) allograft, as well as the importance of thicker grafts in restoring humeral head position and subacromial contact pressure.
From the University of Chicago Pritzker School of Medicine (C.S.L.); Department of Orthopaedic Surgery and Rehabilitation Medicine, UChicago Medicine (M.J., B.S., D.C., A.A.); and Biomechanics Lab, Department of Orthopaedics, University of Illinois at Chicago (F.A.), Chicago, Illinois, U.S.A.

The authors report the following potential conflicts of interest or sources of funding: A.A. reports personal fees from Arthrex, outside the submitted work. Full ICMJE author disclosure forms are available for this article online, as supplementary material.

Received January 22, 2020; accepted May 23, 2020.
Address correspondence to Aravind Athiviraham, M.D., Department of Orthopaedic Surgery and Rehabilitation Medicine, UChicago Medicine, 5841 S. Maryland Ave., MC 3079 Chicago, IL 60637.E-mail: cslee@uchicago.edu (C) 2020 THE AUTHORS. Published by Elsevier Inc. on behalf of the Arthroscopy Association of North America. This is an open access article under the CC BY-NC-ND license (http://creativecommons.org/licenses/by-nc-nd/4.0/). 2666-061X/20105

https://doi.org/10.1016/j.asmr.2020.05.015 


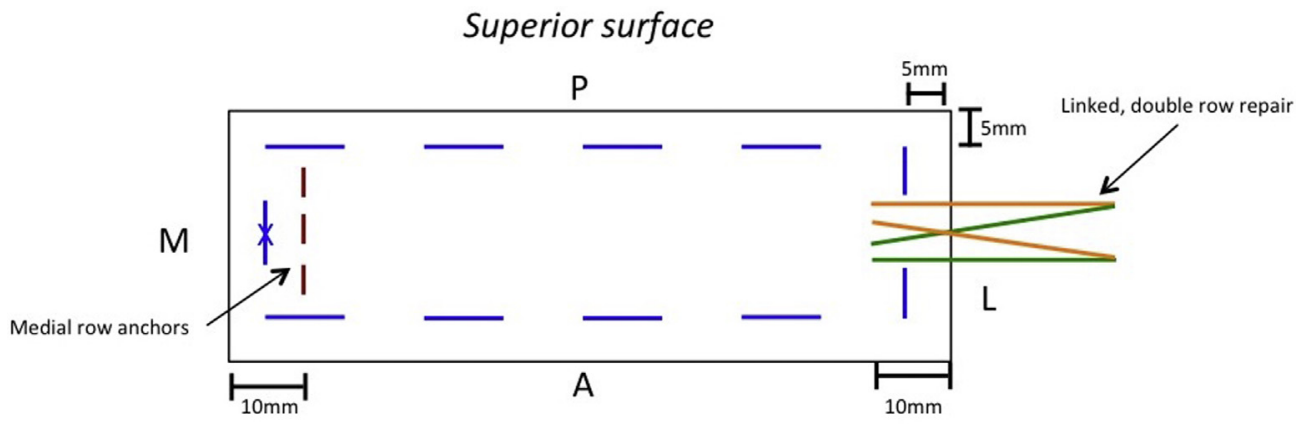

Inferior surface

A

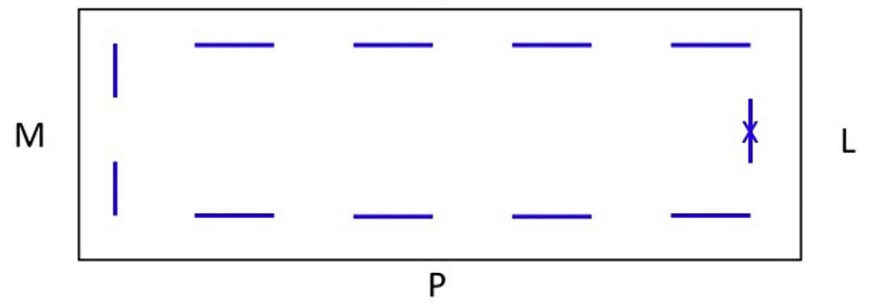

Fig 1. Schematic representation of suture-reinforcement. (A, anterior; $\mathrm{P}$, posterior; $\mathrm{M}$, medial; L, lateral); black color represents graft borders, blue represents suture tape reinforcement, maroon represents medial row anchors on glenoid, and orange and green represent the suture from the 2 anchors used for the linked, double row repair. $\mathrm{X}$ is placed at medial and lateral edges of the HD allograft represents where the 2 suture tapes were tied. (HD, human dermal.)
Despite the encouraging results using FL autograft, allografts remain of clinical interest primarily due to limited donor-site morbidity. Although various allograft materials have been studied, HD allograft remains the most widely used and investigated graft option. In a previous investigation and several retrieval reports, HD allograft has elongated with graft loading, resulting in eventual superior humeral head migration. ${ }^{4}$ Given that the HD allograft is thinner and more susceptible to elastic deformation when compared with FL autograft, various attempts at augmentation have been made, including various subacromial "spacer" techniques, with mixed biomechanical effects on superior humeral head migration and subacromial contact pressure..$^{8,9}$

The purpose of this study was to investigate the potential for a suture tape-reinforcement technique to prevent elongation of a HD allograft with repetitive use in a biomechanical model. We hypothesized that reinforced graft material would demonstrate reduced changes in length, width, and thickness dimensions than nonreinforced grafts, as well as demonstrate greater linear stiffness.

\section{Methods}

The present study is exempt from institutional review board approval. With the use of 8 scapulae and humeri Sawbones models (Sawbones, Vashon Island, WA), the standard SCR was performed using 3 knotless suture anchors placed superomedially on the glenoid at roughly the 10-o'clock, 12-o'clock, and 2-o'clock positions and a linked, double-row repair configuration for fixation laterally. The Sawbones models used provide controlled bone density in this setting for biomechanical testing, as previously described. ${ }^{10,11}$ Eight HD allografts (ArthroFLEX; Arthrex, Naples, FL) were tested: 4 in the native state and 4 using the experimental suture reinforcement. HD allografts were reinforced using suture tape (FiberTape; Arthrex) in a running $360^{\circ}$ fashion around the borders of the HD allograft, maintaining 5 $\mathrm{mm}$ of space from the edges (Figs 1 and 2). Two suture tapes were used per HD allograft and tied together at the medial and lateral edges.

Before the testing of the HD allografts, the suture tape material used for augmentation was loaded to a Materials Testing System (MTS) for 4 trials to quantify its load to failure. This was done to ensure the suture material would maintain its integrity during the final testing of the augmented HD allografts.

Before final testing, HD allografts were measured for length, width, and thickness using a 3-mode digital fractional caliper with a documented accuracy of \pm 0.02 $\mathrm{mm}$. All specimens were affixed to the MTS that allowed for allograft orientation in a longitudinal plane throughout testing (Fig 3). Specimens were preloaded to $10 \mathrm{~N}$ for 60 seconds, after which they were cyclically loaded to $100 \mathrm{~N}$ at a rate of $15 \mathrm{~mm} / \mathrm{s}$ for 30 cycles, consistent with testing conditions in previous studies. $^{12,13} \mathrm{HD}$ allografts were again measured for length, width, and thickness after testing concluded. 


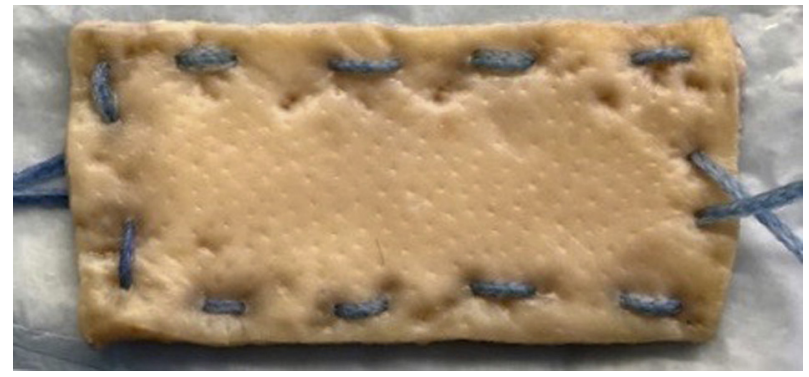

Fig 2. Suture-reinforced HD allograft before testing. The graft is shown before tying of sutures at medial and lateral edges to better illustrate the use of the 2 sutures used. (HD, human dermal.)

Linear stiffness values were calculated by the MTS software and were recorded at cycles 1, 15, and 30.

An a priori sample size calculation was conducted using the established values from the literature regarding native HD allograft elongation following biomechanical testing. ${ }^{4}$ Using the conventional value for alpha of 0.05 and for power set at 0.80, a sample size of 4 specimens per group would be powered to detect a difference in percent change in the range of $3.8 \%$ to $6.4 \%$ or greater. All variables of interest were adequately powered by conventional testing.

The Student $t$ test was used to statistically compare differences in percent change in all dimensions measured for native and reinforced allografts, as well as for linear stiffness values obtained at cycles 1,15 , and 30. A $P$ value of $\leq .05$ was set as significant.

\section{Results}

Initial testing of the suture material yielded a load-tofailure of $895.88 \pm 7.17 \mathrm{~N}$. After dynamic, cyclic loading, reinforced allografts experienced a statistically significant smaller percent change in anterior length $(6.36 \%$ vs $14.50 \%, P=.013)$, posterior length $(6.00 \%$ vs $13.68 \%, P=.002)$, medial width $(5.80 \%$ vs $21.05 \%$, $P=.001)$, lateral width $(5.45 \%$ vs $19.29 \%, P<.001)$, medial thickness $(4.38 \%$ vs $17.93 \%, P=.005)$, central thickness $(7.03 \%$ vs $16.11 \%, P=.026)$, and lateral thickness $(4.55 \%$ vs $20.80 \%, P<.001)$ (Table 1 and Fig 4). Linear stiffness values obtained for reinforced allografts were significantly greater than those for native grafts when measured at cycles $1(21.18 \pm 1.03$ $\mathrm{N} / \mathrm{mm}$ vs $17.69 \pm 1.91 \mathrm{~N} / \mathrm{mm}, P=.02), 15(29.90 \pm$ 1.45 vs $24.93 \pm 2.79, P=.03)$, and $30(32.13 \pm 1.98 \mathrm{~N} /$ $\mathrm{mm}$ vs $25.72 \pm 3.01 \mathrm{~N} / \mathrm{mm}, P=.01$ ) (Table 2 ).

\section{Discussion}

In this biomechanical study, the use of suture tape augmentation for HD allograft material significantly reduced graft elongation in all dimensions. In addition, suture tape augmentation significantly increased the linear stiffness values obtained for the HD allograft material at the beginning, middle, and conclusion of testing. This increase in linear stiffness value of the graft material reflects its ability to withstand elongation and maintain graft thickness.

In a study by Mihata et al., ${ }^{4}$ it was found that SCR using HD allograft restored superior glenohumeral joint force and subacromial contact pressure. However, superior glenohumeral stability was restored only by roughly $50 \%$, and superior humeral head translation remained greater than that of the intact state. This could be due in part to their subsequent finding that the HD allograft experienced approximately 15\% elongation after testing. ${ }^{4}$ Therefore, further investigation into techniques using HD allograft that solve issues posed by graft elongation is warranted and is the focus of the present study. Previous biomechanical studies have largely focused on the effect of graft thickness, capsular continuity, graft tension, and graft material (FL autograft vs HD allograft) for restoration of the glenohumeral joint stability. ${ }^{2-7}$ Makovicka et al. ${ }^{8}$ described a subacromial resurfacing technique that uses the HD allograft as a "spacer" to increase graft thickness and potentially correct acromiohumeral distance. Although this described technique has its potential advantages over traditional SCR with HD allograft, clinical followup data are lacking. To better understand the biomechanical effects of this technique, Curtis et al. ${ }^{9}$

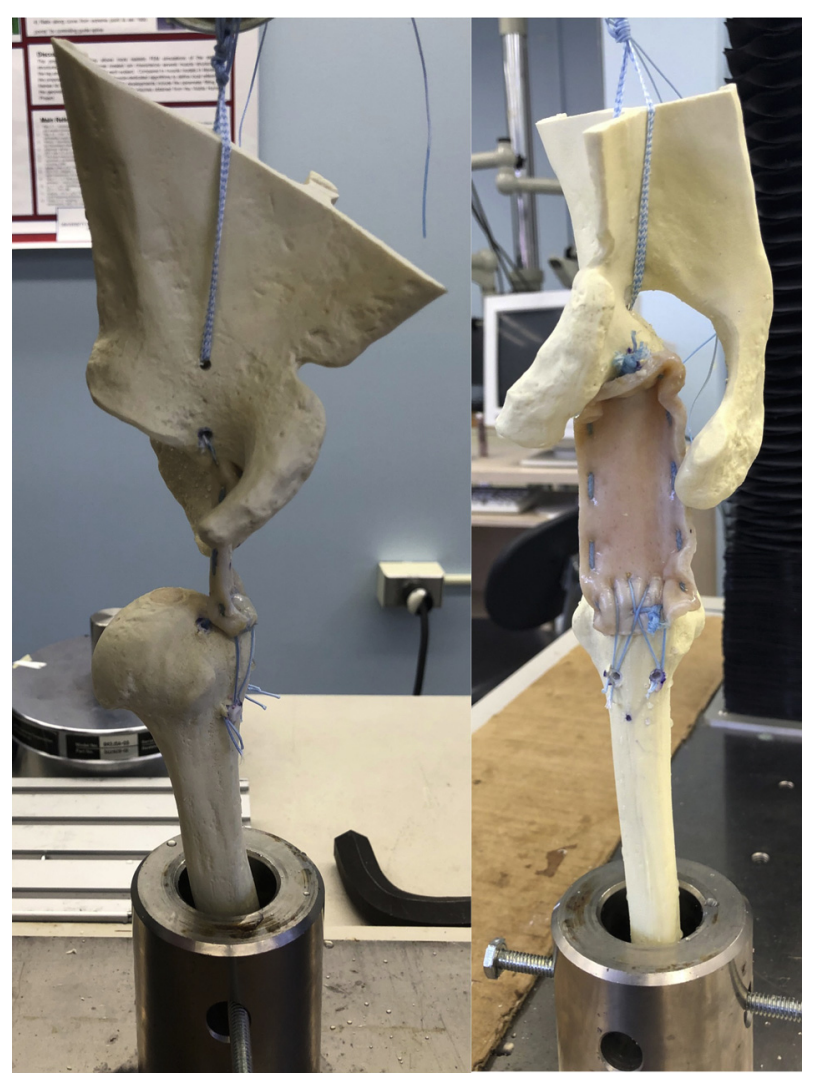

Fig 3. Biomechanical testing setup shown using a reinforced HD allograft; left shows side-view of testing setup; right shows front-view of testing setup. (HD, human dermal.) 
Table 1. Percent Change in Graft Dimensions Following Testing*

\begin{tabular}{|c|c|c|c|c|c|}
\hline & Native & $95 \% \mathrm{CI}$ & Suture-Reinforced & $95 \% \mathrm{CI}$ & $P$ Value \\
\hline Medial width & $-21.05 \pm 2.94 \%$ & $-18.17 \%$ to $-23.93 \%$ & $-5.80 \pm 4.17 \%$ & $-1.71 \%$ to $-9.89 \%$ & .001 \\
\hline Lateral width & $-19.29 \pm 1.64 \%$ & $-17.68 \%$ to $-20.90 \%$ & $-5.45 \pm 3.70 \%$ & $-1.82 \%$ to $-9.08 \%$ & $<.001$ \\
\hline Anterior length & $+14.50 \pm 3.13 \%$ & $+11.43 \%$ to $+17.57 \%$ & $+6.36 \pm 3.50 \%$ & $+2.93 \%$ to $+9.79 \%$ & .013 \\
\hline Posterior length & $+13.68 \pm 2.64 \%$ & $+11.09 \%$ to $+16.27 \%$ & $+6.00 \pm 1.53 \%$ & $+4.50 \%$ to $+7.50 \%$ & .002 \\
\hline Medial thickness & $-17.93 \pm 3.80 \%$ & $-14.21 \%$ to $-21.65 \%$ & $-4.38 \pm 5.12 \%$ & $0.00 \%$ to $-9.40 \%$ & .005 \\
\hline Central thickness & $-16.11 \pm 3.19 \%$ & $-12.98 \%$ to $-19.24 \%$ & $-7.03 \pm 5.28 \%$ & $-1.86 \%$ to $-12.20 \%$ & .026 \\
\hline Lateral thickness & $-20.80 \pm 2.54 \%$ & $-18.31 \%$ to $-23.29 \%$ & $-4.55 \pm 4.55 \%$ & $-0.09 \%$ to $-9.01 \%$ & $<.001$ \\
\hline
\end{tabular}

CI, confidence interval.

*Values demonstrate average \pm standard deviation; $(+)$ values indicate material lengthening and $(-)$ values indicate material shrinkage.

demonstrated that the addition of a subacromial resurfacing technique with use of the HD allograft as a "spacer" resulted in a reduction in superior humeral head migration relative to the intact rotator cuff state and SCR alone. However, the addition of subacromial resurfacing resulted in increased subacromial contact pressures relative to the intact cuff and SCR alone at $30^{\circ}, 60^{\circ}$, and $90^{\circ}$ of shoulder abduction, most likely due to increased material within the subacromial space, and could lead to impingement-related pain with attempted abduction.

Using a different approach, the current study begins to address the issue of superior humeral head migration as a result of graft elongation by investigation of suture tape augmentation of the HD allograft rather than through the use of a "spacer" effect. With this technique, the goal is to not alter the subacromial contact pressure. In a similar effort, Ding et al. ${ }^{14}$ has described a technique for SCR using a "sandwich" patch composed of a polyethylene terephthalate scaffold interspaced between 2 folded layers of FL autograft. ${ }^{14}$ While polyethylene terephthalate has promising applications due to its high tensile strength, clinical data using this technique are lacking. In addition, this procedure is associated with additional cost, is technically challenging, and requires significant increase in operative time. Also, the described "sandwich" patch has not been tested in a biomechanical setting, so it is unknown how it would affect other glenohumeral factors, such as subacromial contact pressure and shoulder range of motion. The goal of the present study is to provide biomechanical proof of concept for a comparatively low-cost suture tape augmentation strategy with the hope of stimulating further biomechanical evaluation.

The strengths of this study included the dynamic, cyclic loading methods used for HD allograft testing, which overcome the weaknesses of other biomechanical studies that have been limited to static measurements. In addition, the current study simulated the standard SCR surgical technique before loading in the MTS. This approach will produce stress on the HD allograft at the graft-fixation sites that resembles the physiologic postoperative state more closely than if the graft material was tested alone. Furthermore, the

\section{Percent Change in Graft Dimensions Following Testing}

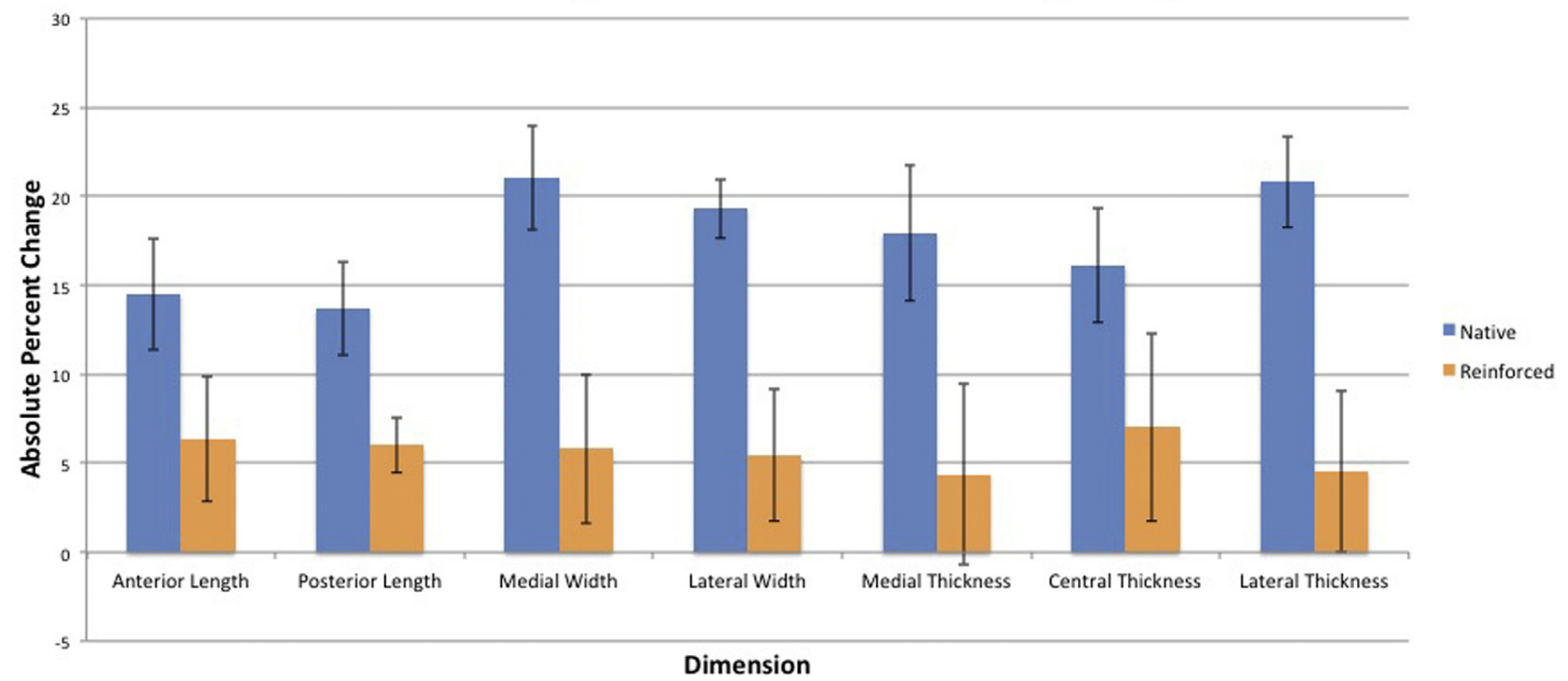

Fig 4. Percent change in graft dimensions following testing. 
Table 2. Linear stiffness (N/mm) Values Obtained for Native Versus Augmented HD Allografts

\begin{tabular}{lccc}
\hline & Native & Suture-Reinforced & $P$ Value \\
\hline Cycle 1 & $17.69 \pm 1.91$ & $21.18 \pm 1.03$ & .024 \\
Cycle 15 & $24.93 \pm 2.79$ & $29.90 \pm 1.45$ & .034 \\
Cycle 30 & $25.72 \pm 3.01$ & $32.13 \pm 1.98$ & .016 \\
\hline
\end{tabular}

HD, human dermal.

*Values demonstrate average \pm standard deviation $(\mathrm{N} / \mathrm{mm})$.

suture tape-augmentation technique described in this study would add minimal operative time or cost. Ultimately, it is felt that this study may serve as a springboard for further studies investigating reinforcement strategies to decrease the elastic deformation and improve strength of HD allograft following cyclic loading, which is particularly relevant in the clinical setting of SCR. Future biomechanical studies should evaluate the differences in superior glenohumeral joint stability and subacromial pressure with and without reinforcement of HD allograft in a cadaveric model.

\section{Limitations}

There were several limitations to this study. First, a Sawbones model rather than a cadaveric model was used, which may limit clinical applicability. However, as the focus of the study was on the graft material properties itself, we did not feel using a cadaveric model would change the results of this study. Of note, we did not experience any anchor pullout or hardware failure during this study. Second, the method of applying linear force across the graft in the longitudinal plane is different than the forces experienced in the graft with dynamic shoulder abduction. Certainly, future biomechanical studies using dynamic, cyclic loading may be able to better clarify if any improvement in superior glenohumeral joint stability and subacromial contact pressure is made with the use of suture tape augmentation. Finally, as with any biomechanical study, we are unable to account for any changes in graft material properties that would occur with biological healing in the clinical setting.

\section{Conclusions}

The suture tape-reinforcement technique described decreased HD allograft elongation, maintained graft thickness, and improved linear stiffness values following uniplanar cyclic loading in a biomechanical Sawbones model.

\section{References}

1. Denard PJ, Brady PC, Adams CR, Tokish JM, Burkhart SS. Preliminary results of arthroscopic superior capsule reconstruction with dermal allograft. Arthroscopy 2018;34:93-99.

2. Mihata T, Lee TQ, Watanabe C, et al. Clinical results of arthroscopic superior capsule reconstruction for irreparable rotator cuff tears. Arthroscopy 2013;29:459-470.

3. Mihata T, McGarry MH, Pirolo JM, Kinoshita M, Lee TQ. Superior capsule reconstruction to restore superior stability in irreparable rotator cuff tears: A biomechanical cadaveric study. Am J Sports Med 2012;40:2248-2255.

4. Mihata T, Bui CNH, Akeda M, et al. A biomechanical cadaveric study comparing superior capsule reconstruction using fascia lata allograft with human dermal allograft for irreparable rotator cuff tear. J Shoulder Elbow Surg 2017;26:2158-2166.

5. Mihata T, McGarry MH, Kahn T, Goldberg I, Neo M, Lee TQ. Biomechanical effect of thickness and tension of fascia lata graft on glenohumeral stability for superior capsule reconstruction in irreparable supraspinatus tears. Arthroscopy 2016;32:418-426.

6. Mihata T, McGarry MH, Kahn T, Goldberg I, Neo M, Lee TQ. Biomechanical role of capsular continuity in superior capsule reconstruction for irreparable tears of the supraspinatus tendon. Am J Sports Med 2016;44: 1423-1430.

7. Mihata T, McGarry MH, Kahn T, Goldberg I, Neo M, Lee TQ. Biomechanical effects of acromioplasty on superior capsule reconstruction for irreparable supraspinatus tendon tears. Am J Sports Med 2016;44:191-197.

8. Makovicka JL, Patel KA, Tokish JM. Superior capsular reconstruction with the addition of an acromial acellular dermal allograft spacer. Arthrosc Tech 2018;7: e1181-e1190.

9. Curtis DM, Lee CS, Qin C, et al. Superior capsule reconstruction with subacromial allograft spacer: Biomechanical cadaveric study of subacromial contact pressure and superior humeral head translation. Arthroscopy 2020;36: 680-686.

10. Douglass NP, Behn AW, Safran MR. Cyclic and Load to Failure Properties of All-Suture Anchors in Synthetic Acetabular and Glenoid Cancellous Bone. Arthroscopy 2017;33:977-985.e5.

11. Robinson S, Krigbaum H, Kramer J, Purviance C, Parrish R, Donahue J. Double row equivalent for rotator cuff repair: A biomechanical analysis of a new technique. J Orthop 2018;15:426-431.

12. Mihata T, Fukuhara T, Jun BJ, Watanabe C, Kinoshita M. Effect of shoulder abduction angle on biomechanical properties of the repaired rotator cuff tendons with 3 types of double-row technique. Am J Sports Med 2011;39: $551-556$.

13. Park JS, McGarry MH, Campbell ST, et al. The optimum tension for bridging sutures in transosseous-equivalent rotator cuff repair: A cadaveric biomechanical study. Am J Sports Med 2015;43:2118-2125.

14. Ding S, Ge Y, Zheng M, et al. Arthroscopic superior capsular reconstruction using "sandwich" patch technique for irreparable rotator cuff tears. Arthrosc Tech 2019;8: e953-e959. 